\title{
Processing capacity in chronic pain patients: A visual event-related potentials study
}

\author{
D.S. Veldhuijzen ${ }^{\mathrm{a}, \mathrm{b}, *}$, J.L. Kenemans ${ }^{\mathrm{a}, \mathrm{c}}$, A.J.M. van Wijck ${ }^{\mathrm{b}}$, B. Olivier ${ }^{\mathrm{a}}$, \\ C.J. Kalkman ${ }^{\text {b }}$, E.R. Volkerts ${ }^{\text {a }}$ \\ ${ }^{a}$ Utrecht Institute for Pharmaceutical Sciences and Rudolf Magnus Institute of Neuroscience, Department of Psychopharmacology, \\ Utrecht University, Utrecht, The Netherlands \\ ${ }^{\mathrm{b}}$ Pain Clinic, Department of Anesthesiology, University Medical Center Utrecht, Utrecht, The Netherlands \\ ${ }^{\mathrm{c}}$ Helmholtz Research Institute, Department of Psychonomics, Utrecht University, Utrecht, The Netherlands
}

Received 7 June 2005; received in revised form 23 November 2005; accepted 5 December 2005

\begin{abstract}
Chronic pain may impair performance on attentional processing capacity tasks. In the present study, event-related potentials were recorded to examine whether pain patients show performance decrements on attentional processing capacity tasks due to shared resources by pain and attention or, alternatively, due to deficits in allocating attentional resources during pain. Fourteen chronic pain patients and thirty age and education matched healthy controls were investigated. An attentional capacity probe task was used in which the difficulty level was manipulated, resulting in an easy and a difficult condition, while task-irrelevant visual probes were presented. These probe-elicited P3 amplitudes were assumed to provide the most pure estimate of processing capacity since they are relatively free from target-related processes. Event-related potentials were recorded from the midline electrodes Fz, $\mathrm{Cz}, \mathrm{Pz}$, and Oz. For the behavioral measures, it was found that pain patients maintained a different speed-accuracy tradeoff. Pain patients showed faster reaction time responses and higher error rates compared to controls. No significant differences were found between pain patients and controls on the primary task. Pain patients differed from controls with respect to amplitudes elicited by task-irrelevant probe stimuli. For healthy controls, the expected decreased amplitude was found for probe stimuli in the difficult compared to the easy task. In contrast, the pain patients did not show decreased probe amplitudes with increasing task load. The data may imply that allocation of attentional resources is deficient in pain patients, instead of attentional capacity.
\end{abstract}

(C) 2005 International Association for the Study of Pain. Published by Elsevier B.V. All rights reserved.

Keywords: Chronic pain; ERPs; Processing capacity; Attention

\section{Introduction}

Chronic pain patients often show attentional deficits, predominantly on tasks that require high attentional demands (Eccleston, 1994; Eccleston and Crombez, 1999; Hart et al., 2000; Dick et al., 2002). Attentional resource models have suggested that these pain-induced performance deficits may be due to the fact that pain and attentional tasks draw upon the same limited atten-

\footnotetext{
* Corresponding author. Tel.: +31 30 2537764; fax: +31 302537387.

E-mail address: D.S.Veldhuijzen@pharm.uu.nl (D.S. Veldhuijzen).
}

tional resources (Eccleston and Crombez, 1999). Pain is regarded as a stressor that automatically attracts resources, which may result in capacity limits being exceeded if task difficulty is high and, subsequently, task performance may decline. Alternatively, an attention switch hypothesis has been proposed in which deficits observed during pain are related to the switching mechanism (Eccleston, 1995), i.e., permanently switching between pain and attention can be assumed to be more difficult when the task is harder. Whether deficits are due to sharing or switching of resources remains to be determined. 
The P3 component of event-related potentials (ERPs) recordings has been extensively studied as a measure of the amount of attentional resources invested in a task (Kok, 2001). The amount of resources that are allocated to a task can be studied with tasks that manipulate difficulty levels, since task difficulty determines the intensity of resource demands (Wickens et al., 1983; Kok, 2001). Two studies have examined the effects of pain on tasks that varied in difficulty in healthy volunteers with experimentally induced pain. Lorenz and Bromm (1997b) showed that task accuracy and P3 amplitude decreased under pain, and Houlihan et al. (2004) demonstrated that this pain-induced decrease was larger in the most difficult task condition. However, no studies have examined attentional processing with varying task difficulty manipulations using ERPs in chronic pain patients.

A commonly used task in the study of attentional resource demands is the probe task (Kenemans et al., 1992; Verbaten et al., 1997; Jonkman et al., 2000; Hoeksma et al., 2004). In this task, secondary task-irrelevant probes are presented against a background of a continuous ongoing primary task of which the difficulty level is manipulated. In general, P3 amplitude elicited by the task-irrelevant probes is thought to reflect a tradeoff in processing demands (Wickens et al., 1983). When the difficulty level of the primary task increases, P3 amplitude to task relevant stimuli increases, reflecting investment of more attentional resources in the difficult task, and P3 amplitude to the task-irrelevant probes decreases, reflecting less spare resources to invest in the secondary task.

In the present study, the question was addressed whether resources are shared by pain and attention, or, alternatively, whether allocating resources during pain is deficient. The P3 amplitude was used to investigate the effects of chronic pain on attentional processing by employing the probe task. With increasing task difficulty, more resources are needed that are assumed to be less available under pain. In this context, we expected a disproportional performance deficit in the difficult task in pain patients compared to controls and/or a disproportional decrease in the probe $\mathrm{P} 3$ in the difficult task in pain patients compared to controls due to less spare capacity.

\section{Methods}

\subsection{Subjects}

Fourteen outpatients ( 4 females and 10 males) with chronic pain and 30 healthy age and education matched controls (15 females and 15 males) participated in the study. Education level was scored according to the 5 categories described in the Wechsler Adult Intelligence Scale (WAIS; Wechsler, 2000). Pain patients were recruited by two pain clinics and healthy controls through local newspaper advertisement. All subjects were paid for their participation. They were all right-handed and had normal or corrected to normal vision.
Patients were included if they had chronic non-cancer pain of moderate to severe intensity (at least $4 \mathrm{~cm}$ on a $10 \mathrm{~cm}$ scale) for at least three months, measured by the pain physician using a Visual Analogue Scale (VAS). Heavy smokers ( $>20$ cigarettes per day), heavy caffeine users $(>5$ cups per day), and subjects that used psychotropic medication, suffered from an alcohol or drug dependence, psychological or psychiatric disorders or severe physical disorders, other than pain for the pain patients, were excluded. The use of paracetamol and/or NSAIDs was not prohibited for pain patients to allow escape medication when needed and to avoid dropouts because of severe pain. Seven of fourteen pain patients used one or more of these allowed analgesic drugs during the study. These patients were asked to continue their analgesic drug at constant dose during the study period. All other (il)licit drugs, apart from oral contraceptives, were prohibited during the total study period. On each test day, compliance was tested using urine drug screening (amphetamines, barbiturates, benzodiazepines, cocaine, morphine, and THC) and a breath alcohol analyzer test. The Medical Ethics Committee of the University Medical Center Utrecht (UMCU) approved the study protocol and written informed consent was obtained from all participants. Procedures were in compliance with the Helsinki Declaration and its latest amendments.

\subsection{Tasks}

In the probe task, difficulty of the primary task was manipulated while probes were presented. The primary task consisted of an easy and a difficult task condition, based on those used in the study of Jonkman et al. (2000). The order of task presentation was counterbalanced across subjects. Each task condition lasted about $10 \mathrm{~min}$ and consisted of two blocks. In each block, 90 task and 90 probe stimuli were presented. Every task stimulus was followed by a probe.

The task stimuli consisted of four different stimuli, which were purple, red, green, and blue rectangles subtending a height of $5.3^{\circ}$ of arc and a width of $4.5^{\circ}$ of arc (adapted from Jonkman et al., 2000). All task stimuli were relevant, since a button press was required after each task stimulus presentation. In the easy task, the subject was instructed to press the right-hand button when a blue rectangle appeared and to press the left-hand button when a rectangle of another color was presented. In each block of the easy condition, 45 blue rectangles and 45 non-blue stimuli (15 red, 15 purple, and 15 green) were presented to ensure equal numbers of left- and right-hand presses. In the difficult task, the subject had to compare each rectangle with the preceding one. When both stimuli were identical, the right-hand button had to be pressed. When the stimulus was different from the preceding one, the left-hand button had to pressed. In each block, there were about an equal number of stimuli of each color (22 or 23), and these were randomized such that equal $(50 \%)$ numbers of right- and left-hand button presses were ensured. The subjects were specifically instructed to respond as quickly as possible, while simultaneously making as few errors as possible. The difficult task could only be correctly executed if the subject kept a running memory of stimuli. This added memory load made the difficult task harder than the easy task. 
The probes consisted of 90 stimuli per block. The probes were chosen according to Verbaten et al. (1997) and included three stimulus types: standards, deviants, and novels. Especially, novels were included since these were presumed to elicit the most pronounced response. Two types of gratings with different spatial frequency and orientation were used, and these were balanced across subjects as standards $(80 \%)$ or deviants $(10 \%)$. Gratings were square-wave, black-on-white, horizontal, high ( 4.8 cycles per degree $(\mathrm{c} / \mathrm{d}))$, or vertical, low $(0.6 \mathrm{c} / \mathrm{d}) \mathrm{spa}-$ tial frequency stimuli. Standards and deviants subtended a height of $5.7^{\circ}$ of arc and a width of $11^{\circ}$ of arc. In line with Hoeksma et al. (2004), novels $(10 \%)$ were unique abstract colored patterns occurring only once during the study. Novels subtended a height of $10.5^{\circ}$ of arc and a width of $11.75^{\circ}$ of arc.

Stimuli were displayed on a monitor positioned approximately one meter from the subject's eyes. Stimulus duration was $100 \mathrm{~ms}$ for the task stimuli, and $924 \mathrm{~ms}$ for the probe stimuli. Interstimulus intervals (ISIs) between stimuli were randomized between 1.7 and $2.3 \mathrm{~s}$. The subjects were told that in between task stimuli other pictures would appear that required attention but no response. They were not specifically instructed which pictures would be presented in between task stimuli, only that these different pictures would alternate with task stimuli. The subjects were required to pay attention to all stimuli, including the pictures that required no response. The examiners closely watched the subjects if they indeed kept focusing on all stimuli, including the probes.

\subsection{Subjective assessment}

The following scales were administered to assess depression and anxiety: Center for Epidemiologic Studies Depression Scale (CES-D; Beekman et al., 1997), Spielberger State-Trait Anxiety scales (STAI; Spielberger, 1983), and five subscales (Depression, Anger, Tension, Vigor, and Fatigue) of the shortened version of the Profile of Mood State (POMS; McNair et al., 1971). Pain intensity was assessed using the Visual Analogue Scale (VAS) from the McGill Pain Questionnaire (MPQ; Melzack, 1975). The left end of the $100 \mathrm{~mm}$ scale was labeled "no pain" and the right "unbearable pain". A global measure of IQ was obtained with the Dutch reading test for adults (NLV; Schmand et al., 1992), adapted from the National Adult Reading Test. Participants read out loud 50 words. Total correctly pronounced words were converted to an estimate of IQ.

\subsection{Procedure}

A training session was held to familiarize the subjects with the procedure and subjects were screened for their ability to perform the difficult task ( $80 \%$ correct criterion). During this session, no EEG was recorded and no novel stimuli were shown. After this training session, the experiments took place. Participants were instructed to abstain from smoking for at least $3 \mathrm{~h}$ prior to the experiment. They were further instructed not to drink caffeinated drinks or alcohol on the day of the experiment. Upon arrival at the laboratory, inclusion and exclusion criteria were verified. After attachment of the electrode cap and EOG electrodes, subjects were seated in a dentist's chair in an acoustically shielded and dimly lighted room. The chair was adjusted so that the subject's head was positioned parallel to the monitor. Instructions for tasks were presented on the monitor. After the instruction, subjects had to perform a short practice session of 20 stimuli. Next, the task was started. Subjects were instructed to move as little as possible during the task and to keep the eyes fixed on the fixation dot on the center of the screen. After each block, the experimenter entered the room and gave instructions for the next block.

\subsection{Electrophysiological recordings}

EEG was recorded from tin midline electrodes $\mathrm{Fz}, \mathrm{Cz}, \mathrm{Pz}$, and $\mathrm{Oz}$, and one additional channel for recording the right mastoid, by means of an Electrocap. The electrodes were referenced to the left mastoid. Horizontal and vertical electrooculogram (EOG) was measured from tin electrodes attached to the outer canthus of each eye and from infra-orbital and supra-orbital electrodes placed in line with the pupil of the left eye. A ground electrode was placed at the middle of the forehead. All electrodes were filled with electrode paste. Electrode impedances were kept below $5 \mathrm{k} \Omega$. Data acquisition was continuous, with a sampling rate of $250 \mathrm{~Hz}$ and a gain of 1000 . Signals were amplified online with a high-pass frequency filter of $0.05 \mathrm{~Hz}$ and a low-pass filter of $100 \mathrm{~Hz}$. Offline, electrophysiological data were rereferenced against linked mastoids. Further, data were filtered with a $30 \mathrm{~Hz} 24 \mathrm{~dB}$ /octave low-pass filter and epoched, starting $100 \mathrm{~ms}$ before stimulus onset and lasting for $800 \mathrm{~ms}$ after stimulus onset.

\subsection{Data analysis}

Reaction times (RTs) were calculated for correct button presses occurring between 150 and $1700 \mathrm{~ms}$ after stimulus onset. Faster or slower responses were discarded. Further, error rates were computed as total errors divided by the sum of errors and correct responses, and omission rates were computed as total omissions divided by the sum of errors, omissions, and correct responses, excluding RTs slower than $150 \mathrm{~ms}$ and faster than $1700 \mathrm{~ms}$.

EEG and EOG data were analyzed offline using Analyzer software (Brain Products $\mathrm{GmbH}$ ). All signals were baseline corrected on the basis of the $100 \mathrm{~ms}$ prestimulus interval. EEG epochs with amplifier blocking, artifacts or flat lines were detected and omitted from further analysis. Ocular artifacts were estimated and subtracted by time domain regression analysis (Gratton et al., 1983). ERPs were sorted for averaging by stimulus type (task stimuli, standards, deviants, and novels), lead ( $\mathrm{Fz}, \mathrm{Cz}, \mathrm{Pz}$, and $\mathrm{Oz}$ ), and group (pain patients, controls). Task ERPs were computed by averaging trials with a correct response. Probe ERPs were computed by averaging only trials in which, in agreement with instructions, no response was given to and the preceding task stimulus was correctly responded to in order to assure attentional investment in the task procedure. The number of trials included in average ERPs was at least 113 for the task stimuli, 13 for the novels, 11 for the deviants, and 85 for the standards. Mean area P3 activity was computed in three $100 \mathrm{~ms}$ segments in the 300-600 window (300-400, 400-500, and 500-600). P3 latency was scored as the global maximum positive amplitude in the 300-600 ms window for the task stimuli on the Pz electrode. Furthermore, exploratively, shorter-latency ERP waves preceding the P3 were also measured. 


\subsection{Statistical analysis}

The data were analyzed using repeated-measures univariate analyses of variance (ANOVA). Behavioral performance measures were conducted with the within-subjects factor Task (2 levels: easy, difficult) and the between-subjects factor Group (2 levels: pain patients, controls). Statistical analysis of mean interval ERP data included the within-subjects factors Task (2 levels: easy, difficult) and Lead (four levels: $\mathrm{Fz}, \mathrm{Cz}, \mathrm{Pz}$, and $\mathrm{Oz}$ ), and the between-subjects factor Group (two levels: pain patients, controls). For the analysis of probe stimuli, the within-subjects factor Stimulus Type (three levels: novels, deviants, and standards) was also included. IQ, measured with the NLV, was used as a covariate for all tests involving the factor Group, since the groups differed on this variable. Statistical analysis of data that was not normally distributed was performed using the Wilcoxon non-parametric test for two related samples. Visual inspection of the data suggested some additional group effects on components preceding the P3. These were investigated in an explorative fashion. Greenhouse-Geisser epsilon and corrected $p$-values are reported where applicable. For all tests, a critical $\alpha$-level of .05 was used. Statistical analyses were performed with SPSS 11.0.1 for Windows.

\section{Results}

Mean age $( \pm \mathrm{SD})$ of the pain patients was 47 (2.3) years, age range: $31-58$ years and for the healthy controls 48 (1.6), age range: $31-60$ years. The mean education level of patients was $3.0(0.3)$ and for controls 3.5 (0.2), corresponding to mean years of education of $12.8(0.9)$ and $14.1(0.7)$, respectively. Despite matching for age and educational level, a significant difference between groups was found for IQ scores measured with the NLV $(F(1,43)=9.17, p<0.004)$, indicating that pain patients had lower IQ scores (mean 94.5) in com- parison to healthy controls (mean 103.7). Mean VAS pain intensity score for the pain patients was $4.8 \mathrm{~cm}$. Table 1 displays pain descriptors for each pain patient separately. All participants scored within the normal range on the depression and anxiety scales.

\subsection{Performance}

As expected, statistical analysis revealed that reaction times increased with increasing task difficulty, confirming the effectiveness of the task difficulty manipulation $(F(1,42)=97.92, p<0.0001)$. A significant main effect of Group was found for reaction times $(F(1,41)=4.68$, $p<0.036)$. In contrast to the expected performance deficits, chronic pain patients had shorter reaction times compared to controls. There was no significant interaction between the effects of Task and Group, indicating that chronic pain patients did not differ from healthy controls on task performance with increasing difficulty.

More errors were made during the difficult compared to the easy task $(F(1,42)=26.00, p<0.0001)$. Further, statistical analysis yielded a significant main effect of Group for error rates $(F(1,41)=5.68, p<0.022)$. Chronic pain patients made significantly more errors than controls. No interaction between Group and Task was found. No effects were found on omission rates. Means and standard errors for behavioral performance data are presented in Table 2.

\subsection{ERP P3 latency}

No differences in P3 latency were found between pain patients and healthy volunteers at the $\mathrm{Pz}$ lead.

Table 1

Pain-related demographic information displayed for each patient separately

\begin{tabular}{|c|c|c|c|c|}
\hline Patient No./Sex/Age (years) & Diagnose & $\begin{array}{l}\text { Duration of pain } \\
\text { complaints (months) }^{\mathrm{a}}\end{array}$ & $\begin{array}{l}\text { VAS pain } \\
\text { intensity }(\mathrm{cm})^{\mathrm{a}}\end{array}$ & Analgesic medication \\
\hline $1 / \mathrm{F} / 31$ & Low back pain & 48 & 2.7 & Paracetamol, nabumeton \\
\hline 2/ M/ 49 & Failed back surgery syndrome & 48 & 1.2 & Paracetamol \\
\hline $3 / \mathrm{M} / 51$ & Radiculopathy L5 & 12 & 5.0 & None \\
\hline 4/ M/ 56 & Low back pain & 84 & 3.4 & None \\
\hline $5 / \mathrm{M} / 57$ & Pain in lower limbs & 36 & 3.1 & None \\
\hline $6 / \mathrm{M} / 58$ & Low back pain & 240 & 3.7 & None \\
\hline $7 / \mathrm{M} / 44$ & $\begin{array}{l}\text { Low back pain combined with } \\
\text { radiculopathy L5 }\end{array}$ & 60 & 5.4 & None \\
\hline $8 / \mathrm{M} / 40$ & Low back pain with neuropathy & 144 & 5.6 & None \\
\hline 9/ F/ 40 & Low back pain & 240 & 4.3 & None \\
\hline $10 / \mathrm{F} / 50$ & Low back pain & 132 & 9.9 & Celecoxib $^{\mathrm{b}}$, Paracetamol, ibuprofen \\
\hline $11 / \mathrm{F} / 49$ & Painful scar in ankle & 120 & 6.8 & Paracetamol \\
\hline $12 / \mathrm{M} / 39$ & Low back pain & 84 & 7.3 & Rofecoxib, paracetamol \\
\hline $13 / \mathrm{M} / 56$ & Radiculopathy S1 right & 48 & 6.6 & Valdecoxib $^{b}$ \\
\hline $14 / \mathrm{M} / 37$ & Painful scar in knee & 24 & 2.8 & Etoricox \\
\hline
\end{tabular}

Abbreviations: F, female; M, male; SI, sacroiliac; S, sacral; and L, lumbar.

${ }^{a}$ Mean VAS score was $4.8 \mathrm{~cm}(\mathrm{SD}=2.3$, range $1.2-9.9 \mathrm{~cm})$ and mean duration of pain complaints was 94.3 months $(\mathrm{SD}=73.4$, range $12-240$ months).

${ }^{\mathrm{b}}$ Medication used on testday. 
Table 2

Mean (SE) reaction time, error rates, and omission rates for the easy and difficult tasks for both the healthy controls and chronic pain patients

\begin{tabular}{lcc}
\hline & Pain patients & Controls \\
\hline Reaction times $(m s)$ & $531.0(28.6)$ & $536.4(15.9)$ \\
Easy & $631.6(28.3)$ & $671.2(18.5)$ \\
Difficult & & \\
Error rates $(\%)$ & $1.6(0.8)$ & $0.5(0.1)$ \\
Easy & $3.3(0.5)$ & $1.9(0.3)$ \\
Difficult & & \\
Omission rates $(\%)$ & $0.7(0.3)$ & $0.8(0.2)$ \\
Easy & $0.9(0.3)$ & $0.3(0.1)$ \\
Difficult & &
\end{tabular}

\subsection{ERP P3 amplitude}

\subsubsection{Task stimuli}

The scalp distribution of the P3 amplitude was consistent with previous reports showing a parietal maximum. No main effects of Group or interactions between Task and Group were found on the P3 amplitude. Statistical analysis revealed a significant interaction between Task and Lead in the $300-400 \mathrm{~ms}$ window $(F(1,42)=9.36, p<0.0001)$. Further analysis showed that significant effects were found at $\mathrm{Fz}$ $(F(1,42)=14.69, p<0.0001)$ and $\mathrm{Cz}(F(1,42)=6.19$, $p<0.017)$, but not at $\mathrm{Pz}$ and $\mathrm{Oz}$. Fig. 1 shows the grand average waveforms for the task stimuli at the $\mathrm{Fz}$ and $\mathrm{Cz}$ leads.

After visual inspection of the grand average waveforms, subsequent statistical tests were performed for the P1 component (100-160 ms) for the task stimuli at the $\mathrm{Pz}$ electrode to examine if groups differed on this component (see Fig. 2). It appeared that, for the Pz P1 component (100-160), amplitude was larger for healthy controls compared to pain patients $(F(1,41)=5.73$, $p<0.021)$.

\subsubsection{Probe stimuli}

In all windows, a significant Task $\times$ Lead $\times$ Group interaction was found $[300-400 \mathrm{~ms}$ window: $F(3,39)=$
5.93, $p<0.010, \varepsilon=.47 ; 400-500 \mathrm{~ms}$ window: $F(3,39)=$ $6.90, \quad p<0.005, \varepsilon=.48$; and $500-600 \mathrm{~ms}$ window $(F(3,39)=5.13, p<0.011, \varepsilon=.59)]$. Further analyses yielded no significant effects in the latest window, however, a significant Task $\times$ Group effect was found in the $300-400 \mathrm{~ms}(F(1,41)=8.69, p<0.005)$ and in the 400 $500 \mathrm{~ms}(F(1,41)=5.83, p<0.020)$ windows at $\mathrm{Oz}$ only. For healthy controls at the $\mathrm{Oz}$ electrode, amplitude in the P3 latency area was smaller in the difficult task compared to the easy task condition, while for the pain patients the reverse pattern was observed (see Fig. 3). Moreover, the difference in this $\mathrm{Oz}$ probe amplitude between the easy and difficult condition was larger for the healthy controls than for the pain patients, though post hoc tests did not yield further significant effects. No significant interaction with Stimulus Type was found, however, exploratively, subsequent analyses were performed to elucidate the most prominent probe. The Task $\times$ Group effects appeared to be most prominent for the novel probes in the $400-500 \mathrm{~ms}(F(3,39)=6.42$, $p<0.004, \varepsilon=.59)$ and $500-600 \mathrm{~ms} \quad(F(3,39)=4.60$, $p<0.015, \varepsilon=.62)$ windows. A significant difference between pain patients and healthy controls for the novel probes on the difficult task condition was found in the $400-500 \mathrm{~ms}(F(1,43)=4.19, p<0.047)$ and $500-600 \mathrm{~ms}$ windows $(F(1,43)=4.14, p<0.048)$, indicating that the amplitude in the $\mathrm{P} 3$ latency range at $\mathrm{Oz}$ was significantly larger in pain patients compared to controls in the difficult but not the easy task condition. Further, subsequent analyses were performed to determine whether the differences between pain patients and controls on the ERPs of the novels probes are related to the effects on error rate in the primary task. In order to do so, we divided the subjects by a median half-split into high-accuracy and low-accuracy performers and entered this as a between-subjects variable in the statistical tests. However, this variable did not affect the results. Grand average waveforms for the novel stimuli at the $\mathrm{Oz}$ electrode are displayed in Fig. 4. For the sake of completeness ERPs at the Pz electrode are also presented since effects were mainly expected on this lead.


Fig. 1. Grand average ERP waveforms to task stimuli in the easy and difficult condition, pooled over groups, on electrodes Fz and Cz. 
$\mathrm{Pz}$

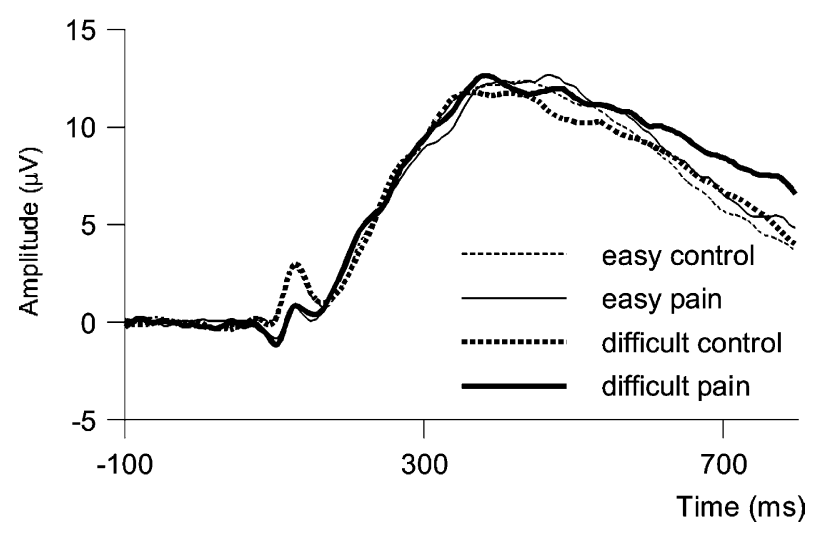

Fig. 2. Grand average waveforms for the task stimuli at the $\mathrm{Pz}$ electrode.

\section{Discussion}

Cognitive involvement in pain processing implies that less attention is available for simultaneous task performance. In the present study, effects of chronic pain on processing capacity were investigated using an ERP probe task with varying task difficulty levels. Moreover, task-irrelevant probe stimuli were presented against the background of the easy and difficult tasks. The secondary task-irrelevant probes were assumed to provide a pure estimate of processing capacity since they are relatively free from target-related processes (Kramer et al., 1995). With increasing task difficulty, more resources are needed that are assumed to be less available under pain. As stated before, we expected a disproportional performance deficit in the difficult task and/or a disproportional decrease in the probe $\mathrm{P} 3$ in the difficult task in pain patients compared to controls due to less spare capacity.

The increasing demands for processing capacity in the difficult task were reflected in increased reaction times and an increase in error rates with increasing task difficulty. These results confirmed the effectiveness of the



Fig. 3. The mean interference scores of Group (pain patients versus controls) and Task (easy versus difficult) for P3 component to the pooled probes on the $\mathrm{Oz}$ electrode. task difficulty manipulation. Furthermore, pain patients differed from the controls both in reaction time and error rate performance. Interestingly, pain patients showed faster responses and higher error rates compared to controls. In essence, these data demonstrate that pain patients adopted a different strategy in the accuracy-speed tradeoff. Further, task performance of patients seems more poorly controlled with more impulsive reactions (e.g., faster and less accurate). Consistent with these findings, Lorenz and Bromm (1997b) also showed that pain reduced accuracy. Moreover, they demonstrated changed response-type dependency of errors and reaction time within a memory search task (false rejections increased and were faster than false acceptances during pain, whereas the opposite occurred during control conditions). However, of importance, the effect of pain did not vary with task difficulty manipulations.

Previous ERP studies to the effects of pain on P3 utilized a standard oddball paradigm, involving simple discrimination of infrequent target and frequent non-target stimuli (Rosenfeld and Kim, 1991; Tandon and Kumar, 1993; Lorenz and Bromm, 1997b, Ozgocmen et al., 2003; Yoldas et al., 2003; Karl et al., 2004). These studies yielded inconclusive results; both increased and decreased P3 amplitudes were found. Noteworthy in this context is the finding that decreased P3 amplitudes under pain can be improved by morphine-induced analgesia (Lorenz et al., 1997a). Further, increased P3 latencies during pain were reported, though not consistently.

Previous studies that applied the probe task found increased P3 amplitudes in the difficult task compared to the easy task with the most prominent effects at the $\mathrm{Pz}$ electrode, indicating investment of more resources in the more difficult task (Wickens et al., 1983; Jonkman et al., 2000; Hoeksma et al., 2004). Consistent with these results, in the present study a significantly increased amplitude was found in the difficult task compared to the easy task condition, however, in particular at the frontocentral leads, and not at Pz. No significant differences were found between pain patients and controls on the primary task. However, pain patients differed from controls on the visual task-irrelevant probe stimuli. It was predicted that P3 amplitudes to the probes would decrease due to increasing task difficulty, and more during pain. For healthy controls, the expected decreased amplitude was found for the probe stimuli in the difficult task compared to the easy task, particularly for the novel probes, however, at $\mathrm{Oz}$ and not as expected at Pz. In contrast, the pain patients did not show a decrease in amplitudes with increasing task load.

The present study showed that P3 latency effects to the task stimuli did not differ between pain patients and controls at Pz. The latency of the P3 component is thought to index stimulus processing speed (Polich and Kok, 1995). The results of the present study are in 



Fig. 4. Grand average waveforms for the novel probes at the Oz electrode.

line with several studies that also found comparable P3 latencies between controls and pain patients (Ozgocmen et al., 2003; Yoldas et al., 2003; Houlihan et al., 2004), although in contrast to some studies which demonstrated increased P3 latencies in pain patients (Tandon and Kumar, 1993; Karl et al., 2004), indicating prolonged stimulus evaluation times during pain. The P3 latency findings may provide an explanation why error rates were higher in pain patients compared to controls. In the present study, stimulus evaluation time was the same for controls and pain patients, however, since patients responded faster to the stimuli, stimulus evaluation was less progressed in time when the response was made, yielding more errors.

Exploratively, effects of pain on ERP components preceding the P3 were examined. The P1 component, an early positive wave around $100 \mathrm{~ms}$, is thought to originate in extrastriate areas and represents early sensory processing. In general, P1 amplitude enlargements are found when stimuli are presented to the attended location (Heinze et al., 1994; Miniussi et al., 1999; Woldorff et al., 2002). Recent studies demonstrate that involuntary as well as voluntary attention may modulate early visual processing as reflected in the P1 component ( $\mathrm{Fu}$ et al., 2005). Interestingly, in the present study $\mathrm{P} 1$ amplitude at the $\mathrm{Pz}$ electrode was larger for controls compared to pain patients, independent of task difficulty. In light of previous findings this might indicate that the stimuli were processed to a lesser extent under pain, however, possible deficits in early processing of stimuli need to be addressed in future studies.

A number of issues need to be considered. First, it should be noted that the difficult task presumably was not so difficult as to reach capacity limits, otherwise, these deficient attentional allocation could have resulted in performance deficits in the difficult task condition, i.e., lower P3 amplitudes to the task stimuli and increased RT and error rates. In future studies, it might be worthwhile to design a more difficult task than the one we used. One possibility would be to trial the task demand and choose a difficult task nearer to ceiling in performance. Second, a possible limitation of this study is the diversity of clinical features of the chronic pain patients. Future studies will need to examine specific subgroups in a larger sample of chronic pain patients in order to determine the generalizability of the present findings.

The main objective of this study was to investigate if effects of pain varied systematically with cognitive load. The controls showed the predicted reciprocal relationship, i.e., increased amplitudes to primary task stimuli and decreased amplitudes to probes due to increasing task difficulty, although at the $\mathrm{Oz}$ and not at the Pz lead. As far as $\mathrm{Oz}$ amplitude represents attentional allocation, the absence of these reciprocal effects in pain patients suggests that pain patients have abnormal attentional allocation. The small insignificant increase in amplitude to probes with increasing load demonstrated that pain patients do not suffer from shortage of capacity of resources. This interpretation is strengthened since primary task performance was not affected by pain. Apparently, pain patients did not need to 'borrow' resources that were used to process the probe stimuli in order to efficiently process the task stimuli. Rather, the data imply that allocation of attentional resources is deficient in pain patients. It appears that the task-irrelevant stimuli impose greater distraction on pain patients than on controls as indicated by higher ERP P3 amplitude elicited by novels and by more poorly controlled and more impulsive reactions (e.g., faster and less accurate) to subsequent task stimuli. These findings point to deficits in disengagement of attention. Consistent with these findings, recent studies on the attentional demand of pain demonstrated that disengagement of attention was deteriorated when experimentally induced pain was anticipated but did not occur (Van Damme et al., 2002, $2004 a, b)$. Our present study demonstrated that problems of disengagement of attention are not only found in anticipation of pain, but also when continuously in 
pain, and not only to pain-related cues, but also to stimulus processing of novel infrequent occurring stimuli. These findings can be explained with the recently proposed model of hypervigilance as a feature of chronic pain (Crombez et al., 2005). These authors argue that because hypervigilance is unintentional and efficient, pain processing may interfere with task stimuli processing despite being counterproductive for task performance. Hypervigilance may render chronic pain patients more vulnerable to distraction, especially towards novel stimuli. Further, it should be noted that difficulties in disengagement from pain are mediated by factors such as catastrophizing or fearful anticipation of pain (e.g., Van Damme et al., 2002, 2004a,b; Crombez et al., 2005).

The results found in this study are moreover in line with the findings of Jonkman et al. (2000) and Hoeksma et al. (2004). These authors examined processing capacity in autistic subjects and also found that attentional allocation, but not capacity, was affected. They argued that this indicated impairment in flexibility. This could also be the case for pain patients, however, this should be addressed in future studies.

\section{Acknowledgement}

This research was supported by internal funds only and there were no competing interests.

\section{References}

Beekman AT, Deeg DJ, Van Limbeek J, Braam AW, De Vries MZ, Van Tilburg W. Criterion validity of the Center for Epidemiologic Studies Depression scale (CES-D): results from a community-based sample of older subjects in The Netherlands. Psychol Med 1997;27:231-5.

Crombez G, Van Damme S, Eccleston C. Hypervigilance to pain: an experimental and clinical analysis. Pain 2005;116:4-7.

Dick B, Eccleston C, Crombez G. Attentional functioning in fibromyalgia, rheumatoid arthritis, and musculoskeletal pain patients. Arthritis Rheum 2002;47:639-44.

Eccleston C. Chronic pain and attention: a cognitive approach. Br J Clin Psychol 1994;33:535-47.

Eccleston C. Chronic pain and distraction: an experimental investigation into the role of sustained and shifting attention in the processing of chronic persistent pain. Behav Res Ther 1995;33:391-405.

Eccleston C, Crombez G. Pain demands attention: a cognitive-affective model of the interruptive function of pain. Psychol Bull 1999; 125:356-66.

$\mathrm{Fu}$ S, Greenwood PM, Parasuraman R. Brain mechanisms of involuntary visuospatial attention: an event-related potential study. Hum Brain Mapp 2005;25:378-90.

Gratton G, Coles MG, Donchin E. A new method for off-line removal of ocular artifact. Electroencephalogr Clin Neurophysiol 1983;55:468-84.

Hart RP, Martelli MF, Zasler ND. Chronic pain and neuropsychological functioning. Neuropsychol Rev 2000;10:131-49.
Heinze HJ, Mangun GR, Burchert W, Hinrichs H, Scholz M, Munte $\mathrm{TF}$, et al. Combined spatial and temporal imaging of brain activity during visual selective attention in humans. Nature 1994:372:543-6.

Hoeksma MR, Kemner C, Verbaten MN, van Engeland H. Processing capacity in children and adolescents with pervasive developmental disorders. J Autism Dev Disord 2004;34:341-54.

Houlihan ME, McGrath PJ, Connelly JF, Stroink G, Finley GA, Dick $\mathrm{B}$, et al. Assessing the effect of pain on demands for attentional resources using ERP's. Int J Psychophysiol 2004;51:181-7.

Jonkman LM, Kemner C, Verbaten MN, Van Engeland H, Camfferman G, Buitelaar JK, et al. Attentional capacity, a probe ERP study: differences between children with attention-deficit hyperactivity disorder and normal control children and effects of methylphenidate. Psychophysiology 2000;37:334-46.

Karl A, Diers M, Flor H. P300-amplitudes in upper limb amputees with and without phantom limb pain in a visual oddball paradigm. Pain 2004;110:40-8

Kenemans JL, Verbaten MN, Melis CJ, Slangen JL. Visual stimulus change and the orienting reaction: event-related potential evidence for a two-stage process. Biol Psychol 1992;33:97-114.

Kok A. On the utility of P3 amplitude as a measure of processing capacity. Psychophysiology 2001;38:557-77.

Kramer AF, Trejo LJ, Humphrey D. Assessment of mental workload with task-irrelevant auditory probes. Biol Psychol 1995;40:83-100.

Lorenz J, Beck H, Bromm B. Cognitive performance, mood and experimental pain before and during morphine-induced analgesia in patients with chronic non-malignant pain. Pain 1997a;73:369-75.

Lorenz J, Bromm B. Event-related potential correlates of interference between cognitive performance and tonic experimental pain. Psychophysiology 1997b;34:436-45.

McNair DM, Lorr M, Droppleman LF. Edits manual for the profile of mood states. San Diego, USA: Educational and Industrial Testing Service; 1971.

Melzack R. The McGill pain questionnaire: major properties and scoring methods. Pain 1975;1:277-99.

Miniussi C, Wilding EL, Coull JT, Nobre AC. Orienting attention in time. Modulation of brain potentials. Brain 1999;122:1507-18.

Ozgocmen S, Yoldas T, Kamanli A, Yildizhan H, Yigiter R, Ardicoglu O. Auditory P300 event related potentials and serotonin reuptake inhibitor treatment in patients with fibromyalgia. Ann Rheum Dis 2003:62:551-5.

Polich J, Kok A. Cognitive and biological determinants of P300: an integrative review. Biol Psychol 1995;41:103-46.

Rosenfeld JP, Kim M. Ongoing pain as a mental workload indexed by P300 depression: discrimination of real and feigned pain conditions. Psychophysiology 1991;28:336-43.

Schmand B, Lindeboom J, van Jarskamp F. Manual for the Dutch reading test for adults. Lisse, the Netherlands: Swets \& Zeitlinger BV; 1992.

Spielberger CD. Manual for the state-trait anxiety inventory (STAI-form Y). Palo Alto, CA, USA: Consulting Psychologists Press; 1983.

Tandon OP, Kumar S. P3 event related cerebral evoked potential in chronic pain patients. Indian J Physiol Pharmacol 1993;37:51-5.

Van Damme S, Crombez G, Eccleston C. Retarded disengagement from pain cues: the effects of pain catastrophizing and pain expectancy. Pain 2002;100:111-8.

Van Damme S, Crombez G, Eccleston C. Disengagement from pain: the role of catastrophic thinking about pain. Pain 2004a; $107: 70-6$

Van Damme S, Crombez G, Eccleston C. The anticipation of pain modulates spatial attention: evidence for pain-specificity in highpain catastrophizers. Pain 2004b;111:392-9.

Verbaten MN, Huyben MA, Kemner C. Processing capacity and the frontal P3. Int J Psychophysiol 1997;25:237-48. 
Wechsler D. Manual for the wechsler adult intelligence scale. New York-III: psychological corporation. Dutch translation. Lisse, the Netherlands: Swets \& Zeitlinger BV; 2000.

Wickens C, Kramer A, Vanasse L, Donchin E. Performance of concurrent tasks: a psychophysiological analysis of the reciprocity of information-processing resources. Science 1983;221:1080-2.
Woldorff MG, Liotti M, Seabolt M, Busse L, Lancaster JL, Fox PT. The temporal dynamics of the effects in occipital cortex of visualspatial selective attention. Brain Res Cogn Brain Res 2002;15:1-15.

Yoldas T, Ozgocmen S, Yildizhan H, Yigiter R, Ulvi H, Ardicoglu O. Auditory p300 event-related potentials in fibromyalgia patients. Yonsei Med J 2003;44:89-93. 